Eur. J. Clin. Chem. Clin. Biochem.

Vol. 29, 1991, pp. $417-424$

(C) 1991 Walter de Gruyter \& Co.

Berlin - New York

\title{
Mean and Variance Rules are More Powerful or Selective than Quality Control Rules Based on Individual Values
}

\author{
By $K$. Linnet \\ Department of Clinical Chemistry, Rigshospitalet, Copenhagen, Denmark
}

(Rcceived July 9, 1990/April 12, 1991)

Summary: Quality control rules based on individual values are compared with mean and variance rules using theoretical computations and simulations. Simple $\left(1_{3 s}\right)$ and combined individual value rules, e. g. a $1_{3 s} / 2_{2 s} / 4_{1 s} / 6_{\dot{x}}$ rule, are all less powerful for detection of shifts of location than a mean rule, given identical type I errors. The mean rule is also more robust towards non-normality of data distributions. In most cases, the variance rule has more power towards increased scatter than individual value rules, and it always has the highest selectivity. Thus, the simple computations that are required for derivation of the mean and variance result in increased power or selectivity. In particular, in the computerization of quality control, the traditional mean and variance rules are preferable to more or less complicated "multi-rules" proposed for computerized quality control.

\section{Introduction}

Control rules based on the mean and range were originally transferred from industry to clinical chemistry without modifications (1). Levey \& Jennings chose two control samples per run, but later Henry \& Segalove (2) proposed the use of only a single control in a run. Subsequently, it has become customary to operate with control rules based on individual values, even in cases with more than one control observation per run (3). To begin with, simple control rules were usẹd, e.g., rejection if at least one control value deviated by more than three standard deviations from the target value (often called a $1_{3 s}$ rule), but more sophisticated combined rules or multi-rules were introduced later, in order to increase the power $(3-6)$. Individual value rules have probably gained popularity because they are easy to use. Once the control limits have been established, no further calculations are necessary, in contrast to mean and range (variance) rules. Today, however, computations are easily performed with laboratory computers, and the application of mean and variance rules is practical. Against this background, the power, selectivity, and robustness towards non-normality of the two principles were compared.

\section{The Principle of Quality Control Rules}

A quality control rule is a statistical test of the null hypothesis that the analysis is in control. In this state, the control values are distributed about the target value with a certain dispersion characteristic for the analytical procedure. The process may go out of control because of a fixed location shift or because of an increased scatter. For mnemotechnical reasons, a control rule may be symbolized as $A_{L}$, where $A$ is a number or some aggregate measure of the control values, which shall exceed the limit $L$ to signal a rejection (3). For example, a control rule implicating a rejection, if at least one control observation deviates by more than three standard deviations from the target value, may be denoted a $1_{3 s}$ rule. Tables 1 and 2 display some commonly used rules for detecting location shifts and increased scatter, respectively. A quality control rule is subject to two kinds of errors: a type I error $(\alpha)$, which consists of declaring the process out of control, although no real errors are present, and a type II error ( $\beta$ ), which is assurance of an in-control state, even though the process actually is out of control. Ideally, the probabilities of these errors should both be zero, but in practice they are of a certain magnitude. The grater the number of 
Tab. 1. Control rules primarily for detection of shift of location

\begin{tabular}{|c|c|}
\hline Symbol $\left(A_{L}\right)$ & Description \\
\hline $1_{3 s}$ & $\begin{array}{l}\text { Simple individual value rule. } \\
\text { Rejection if one control deviates by more than } \\
\text { three standard deviations from the target } \\
\text { value. }\end{array}$ \\
\hline $1_{3 s} / 2_{2 s}$ & $\begin{array}{l}\text { Combined individual value rule. } \\
\text { Rejection if one control deviates by more than } \\
\text { three standard deviations from the target } \\
\text { value, } \\
\text { or } \\
\text { if two controls deviate by more than two } \\
\text { standard deviations in the same direction } \\
\text { from the target value. }\end{array}$ \\
\hline $1_{3 s} / 2_{2 s} / 4_{1 s} / 6_{\bar{x}}$ & $\begin{array}{l}\text { Combined individual value rule. } \\
\text { Rejection if one control deviates by more than } \\
\text { three standard deviations, } \\
\text { or } \\
\text { two controls deviate by more than two stand- } \\
\text { ard deviations, } \\
\text { or } \\
\text { four controls deviate by more than one stand- } \\
\text { ard deviation from the target value, } \\
\text { or } \\
\text { six controls all are located on the same side } \\
\text { of the target value. }\end{array}$ \\
\hline $\bar{x} \bar{r}_{L}$ & $\begin{array}{l}\text { Mean rule. } \\
\text { Rejection if the mean of the controls, } \\
\qquad \bar{x} \bar{r}=\sum x_{i} / N, \\
\text { deviates by more than } L \text { from the target value. }\end{array}$ \\
\hline $\bar{x} \bar{r}_{L 3} / 2 \bar{x} \bar{r}_{L 2}$ & $\begin{array}{l}\text { Combined mean rule. } \\
\text { Rejection if the mean of the controls of the } \\
\text { current run deviates by more than } L 3 \text {, } \\
\text { or } \\
\text { the means of the preceding and current runs } \\
\text { both deviate by more than } L 2 \text { (in the same } \\
\text { direction) from the target value. }\end{array}$ \\
\hline $\bar{x} \bar{r}_{L 3} / 2 \bar{x} \bar{r}_{L 2} / 5 \bar{x} \bar{r}_{\bar{x}}$ & $\begin{array}{l}\text { Combined mean rule. } \\
\text { Rejection if the mean of the current run de- } \\
\text { viates by more than } L 3 \text {, } \\
\text { or } \\
\text { the means of the preceding and current runs } \\
\text { both deviate by more than } L 2 \text { (in the same } \\
\text { direction) from the target value, } \\
\text { or } \\
\text { the latest five means are located on the same } \\
\text { side of the target value. }\end{array}$ \\
\hline $\bar{x} \bar{r}_{L 3} / \bar{x} \bar{r}(5)_{L 2}$ & $\begin{array}{l}\text { Mean rule combined with a moving average } \\
\text { rule. } \\
\text { Rejection if the mean of the current run de- } \\
\text { viates by more than } L 3 \text {, } \\
\text { or } \\
\text { the mean value of all controls in the latest } \\
\text { five runs (the moving average) deviates by } \\
\text { more than } L 2 \text { from the target value. }\end{array}$ \\
\hline
\end{tabular}

controls per run, the smaller the errors can be made. Some reasonable compromise between error levels and costs has to be made.
Tab. 2. Control rules primarily for detection of increased scatter

\begin{tabular}{ll}
\hline Symbol $\left(A_{L}\right)$ & Description \\
\hline$R_{4 s}$ & Range rule: \\
& a) Individual value modification: \\
& Rejection if one control is smaller than the \\
& target value minus two standard deviations \\
& and one exceeds the target value plus two \\
& standard deviations. \\
& b) Original version: \\
& Rejection if the difference between the \\
& maximum and minimum control value ex- \\
& ceeds four standard deviations.
\end{tabular}

$s r_{L}^{2}$

Variance rule.

Rejection if the squared standard deviation of the distribution of controls,

$$
s r^{2}=\sum\left(x_{i}-\bar{x} \bar{r}\right)^{2} /(N-1),
$$

exceed the limit

$$
L=\chi_{(1-\alpha)(N-1)}^{2}\left[s^{2} /(N-1)\right]
$$

The complement to the type II error $(1-\beta)$, the power, is the probability of detecting an out-of-control state when the process really is out of control. A comparison of the powers of two quality control rules is only fair, if the type I error levels are identical. Otherwise a comparison is biased. In the following sections, the powers of the mean and variance rules will be compared with those of various individual value control rules. Control rules limited to a single analytical run as well as rules covering several runs are considered. The comparisons are based on theoretical computations or simulations as specified in the Appendix.

\section{Shift of Location: Comparison of the Powers of the Mean Rule and Individual Value Rules}

Power curves of the $1_{3 s}$ and $\bar{x} \bar{r}_{L}$ rules (tab. 1) for detection of systematic errors are shown in figure 1 for $N=2$ and 6 controls per run, illustrating situations with relatively few and relatively many controls, respectively. In model studies as here, $s$ and $\vec{x}$ are identical to the true parameter values $\sigma$ and $\mu$, re= spectively. However, in real situations $\sigma$ and $\mu$ are always unknown, and only estimates are available from the initial method evaluation study. Therefore, the notations $s$ and $\bar{x}$ have been used throughout in this paper. The $\bar{x} \bar{r}_{L}$ rule signifies a rejection if the - mean of the control values in a run $(\bar{x} \bar{r})$ deviates by more than $L$ from the target value $(\bar{x})$. The type I 


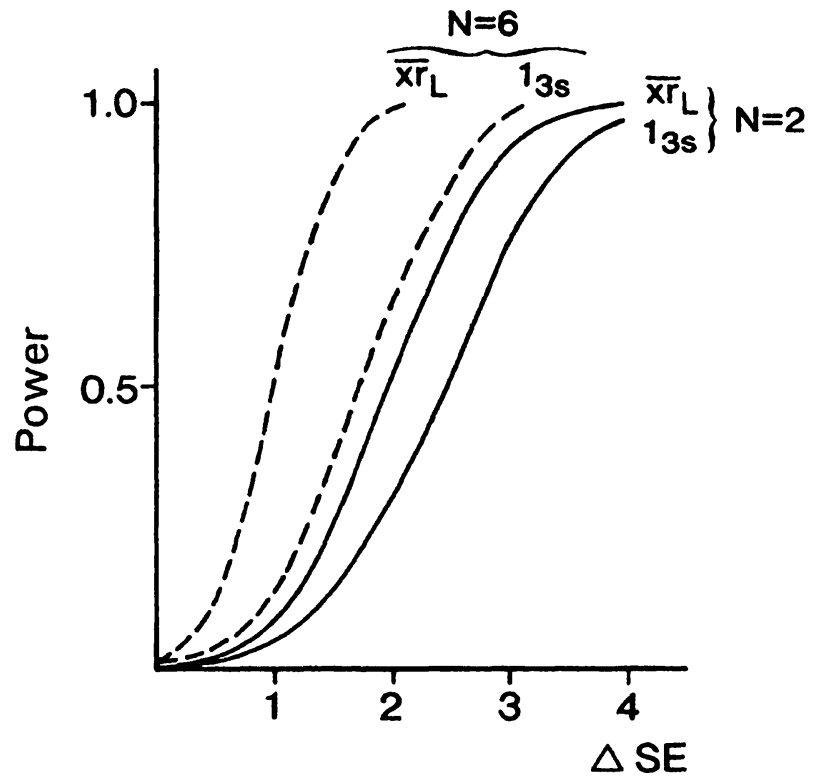

Fig. 1. Power curves for the $1_{3 s}$ and $\bar{x} \bar{r}_{L}$ rules. $N=2$ or 6 controls per run.

$\Delta \mathrm{SE}$ is the location shift expressed in standard deviation (s) units.

error $(\alpha)$ of the $1_{3 s}$ rule changes from 0.00540 to 0.01609 when $N$ increases from 2 to 6 . By appropriate selections of the $L$ values, the type I error of the mean rule has been fixed at a corresponding level for each $N$ (Appendix). The power of the $\bar{x} \bar{r}_{L}$ rule is up to 1.5 $(N=2)-4(N=6)$ times that of the $1_{3 s}$ rule.

Rules based on a combination of individual values are frequently used, e.g. a $1_{3 s} / 2_{2 s}$ rule (5) (tab. 1). The power curves (fig. 2) of this rule and the $\bar{x} \bar{r}_{L}$ rule, again with equivalent type I errors for $N=2$ and 6 , show that $\bar{x} \bar{r}_{L}$ has up to $1.3-2.5$ times to power of the combined rule. For $N=6$ controls per run, a multi-rule can be devised, e.g. $1_{3 s} / 2_{2 s} / 4_{1 s} / 6_{\bar{x}}$ (tab. 1). $\sigma_{x}$ means 6 control values on the same side of the target value. A comparison of this multi-rule and the $\bar{x} \bar{r}_{L}$ rule, given equivalent type I errors $(\alpha=0.0535)$, reveals that $\bar{x} \bar{r}_{L}$ is superior with a power up to 1.4 times that of the multi-rule (fig. 3).

\section{Increased Sçatter: Comparison of Powers of Variance and Individual Value Rules}

The range rule $R_{4 s}$ detects increased scatter (tab. 2). In the original version this rule is not an individual value rule, because the difference between the maximum and minimum control value $(=R)$ in a run has to be computed and compared with $4 s$. The individual value modification consists of defining a reject signal as the simultaneous occurrence in a run of one control

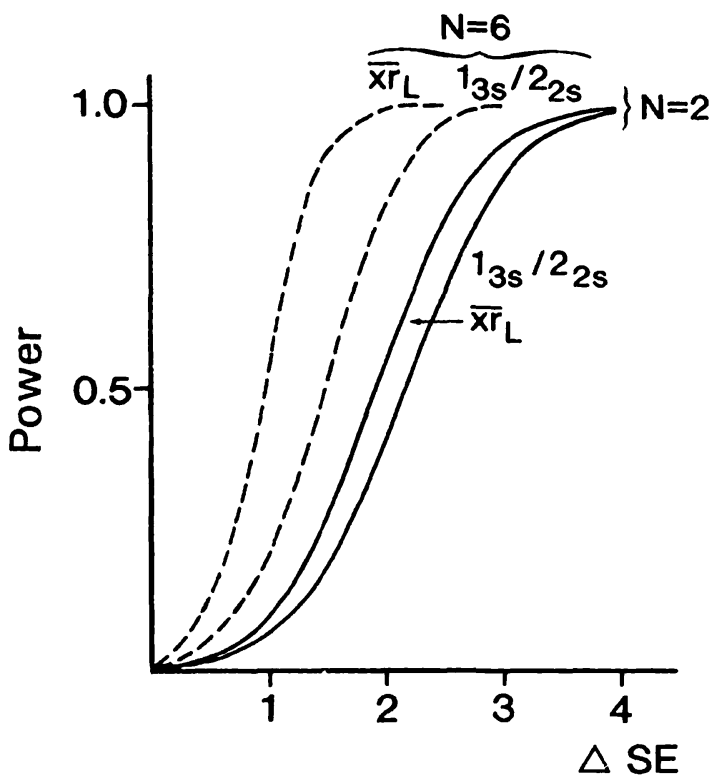

Fig. 2. Power curves for $1_{3 s} / 2_{2 s}$ and $\bar{x} \bar{r}_{L}$ rules. $\Delta \mathrm{SE}$ is the location shift expressed in standard deviation (s) units.

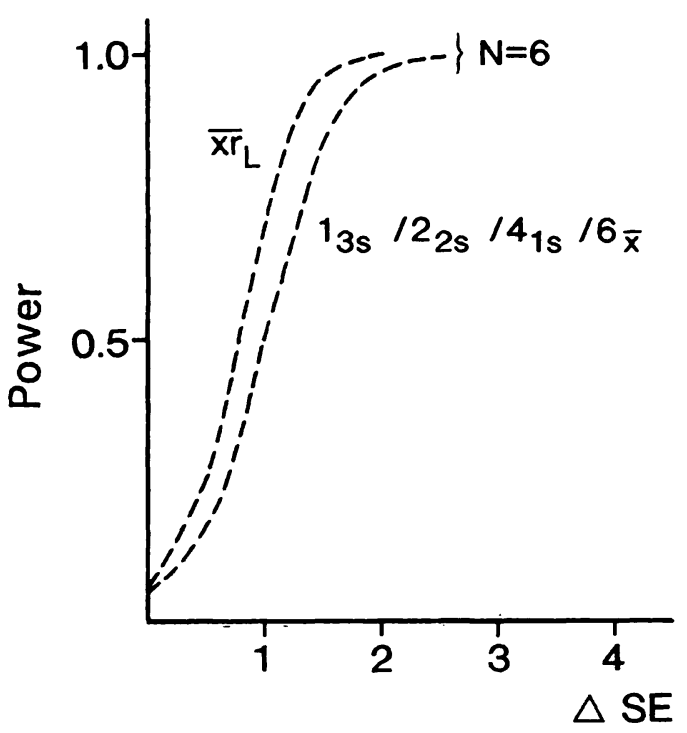

Fig. 3. Power curves for the $1_{3 s} / 2_{2 s} / 4_{1 s} / 6_{\bar{x}}$ and $\bar{x} \bar{r}_{L}$ rules. $\Delta \mathrm{SE}$ is the location shift expressed in standard deviation (s) units.

value below the $-2 s$ limit and one value above the $+2 s$ limit. This slight modification actually decreases the power to less than half the value of the original rule $(N=2)$ (fig. 4$)$. In this comparison the limit $4 s$ has been modified to $4.636 s$ for the original range rule to ensure equivalent type I errors $(\alpha=0.001035)$.

For $N=2$ the original range rule and a variance rule are equally efficient, i. e. given the same type I errors, the power curves coincide. But for $N$ greater than 2, the variance rule $\left(s r_{L}^{2}\right)(\operatorname{tab} .2)$ is the most powerful. 


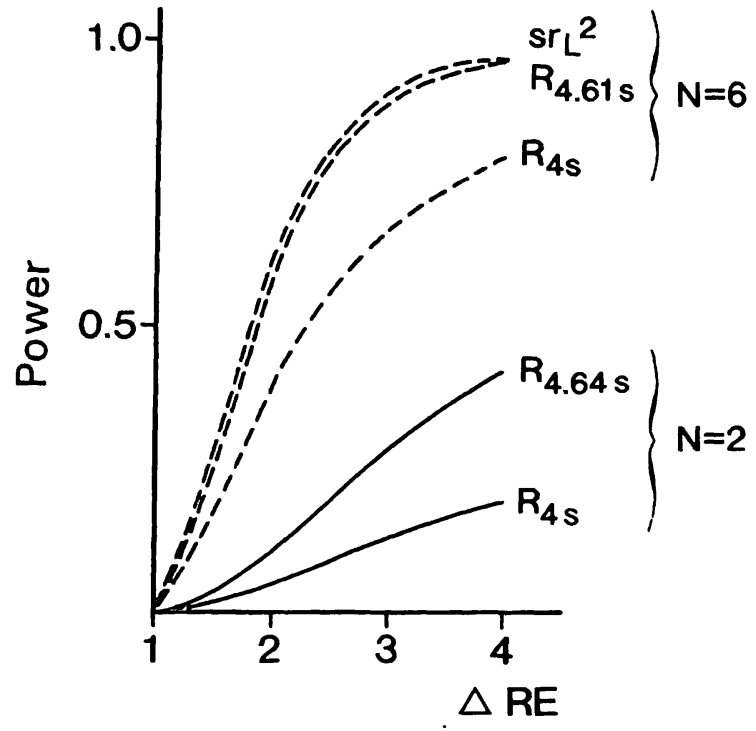

Fig. 4. Power curves for the individual value range rule $\left(R_{4 s}\right)$, the original range rule with equivalent type $I$ errors $\left(R_{4.64 s}(N=2)\right.$ and $\left.R_{4.61 s}(N=6)\right)$, and the variance rule $s r_{L}^{2}(N=6)$.

$\triangle R E$ is the increased scatter expressed as multiples of the standard deviation $s$.

The squared standard deviation of the distribution of observed control values in a run

$$
s r^{2}=\sum\left(x_{i}-\bar{x} \vec{r}\right)^{2} /(N-1)
$$

is compared with the limit

$$
L=\chi_{(1-\alpha)(N-1)}^{2}\left[s^{2} /(N-1)\right],
$$

where $\chi_{(1-\alpha)(N-1)}^{2}$ is the $(1-\alpha)$ fractile of the $\chi^{2}$ distribution with $(N-1)$ degrees of freedom, and $s$ is the estimate of the within-run standard deviation obtained in the initial phase of method evaluation. Provided that $s$ is estimated from a reasonably large number of runs $(>=20-30)$ and the distribution of control values is Gaussian, the type I error of this rule equals $\alpha$. For $N=6$ controls per run, the variance rule is better than both types of range rules, with the greatest difference corresponding to a factor 1.5 compared with the individual value modification ( $\alpha=0.01422$ for all rules) (fig. 4).

The $1_{3 s}$ rule, considered in the previous section as a control rule for detection of increased scatter, is also capable of revealing random errors (fig. 5). For $N=2$ this rule is actually more powerful than the variance (or original range) rule, whereas the contrary holds true for $N=6$. Thus, for the first time an example is presented, in which an individual value rule outperforms a "traditional rule from industry, at least for some values of $N$. However, as analysed in more detail in the next section, the variance rule has the advantage (for all $N$ ) of being more specific than the $1_{3 s}$ rule for detection of increased scatter.

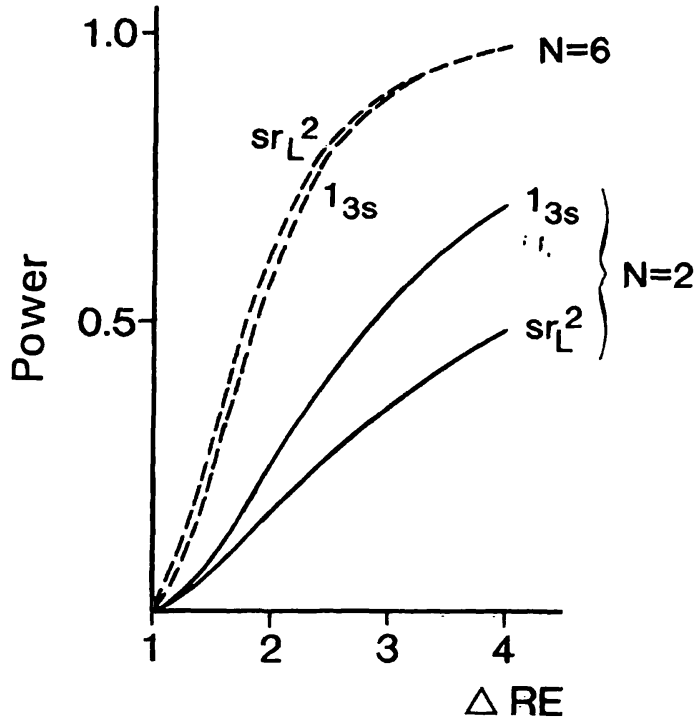

Fig. 5. Power curves for the $1_{3 s}$ and $s r_{L}^{2}$ rules.

$\triangle \mathrm{RE}$ is the increased scatter expressed as multiples of the standard deviation $s$.

\section{Selectivity of Control Rules Towards Shift of Location or Increased Scatter}

If a quality control rule responds selectively to either systematic or random errors, a reject signal indicates the type of error that is present, and the search for the underlying cause of error is facilitated. To characterize the selectivity of control rules, the concepts specificity and sensitivity from the area of diagnostic tests can be useful. The definitions are:

$$
\begin{aligned}
& \text { Sensitivity }=N(\mathrm{TP}) /[N(\mathrm{TP})+N(\mathrm{FN})] \\
& \text { Specificity }=N(\mathrm{TN}) /[N(\mathrm{TN})+N(\mathrm{FP})]
\end{aligned}
$$

where $N(\mathrm{TP})$ is the number of true positive results, $N(\mathrm{FN})$ is the number of false negatives, $N(\mathrm{TN})$ is the number of true negatives, and $N(\mathrm{FP})$ is the number of false positives. The $\bar{x} \bar{r}_{L}$ and $2_{2 s}$ rules are intended for detection of location shift, and the power can be regarded as the sensitivity. Reject signals caused by increased scatter may be interpreted as false positives and, accordingly, the proportion of runs without a reject signal in the presence of increased scatter becomes the specificity. In the comparison of specificitiès of the $\bar{x} \bar{r}_{L}$ and $2_{2 \text { s }}$ rules, the limit $L$ has been adjusted so that the type I errors are identical. The specificity of the $2_{2 s}$ rule is greater for $N=2$, whereas the reverse is true for $N=6$ (fig. 6). However, the apparent advantage of the $2_{2 s}$ rule for $N=2$ is balanced by a lower sensitivity towards systematic errors than that of the mean rule, given equivalent type I errors (e. g., 0.25 versus 0.33 for a location shift of $2 s$ ). Further- more, $2_{2 s}$ is seldom used alone, but rather as a part of the $1_{3 s} / 2_{2 s}$ rule, and this combination has a very low specificity. 


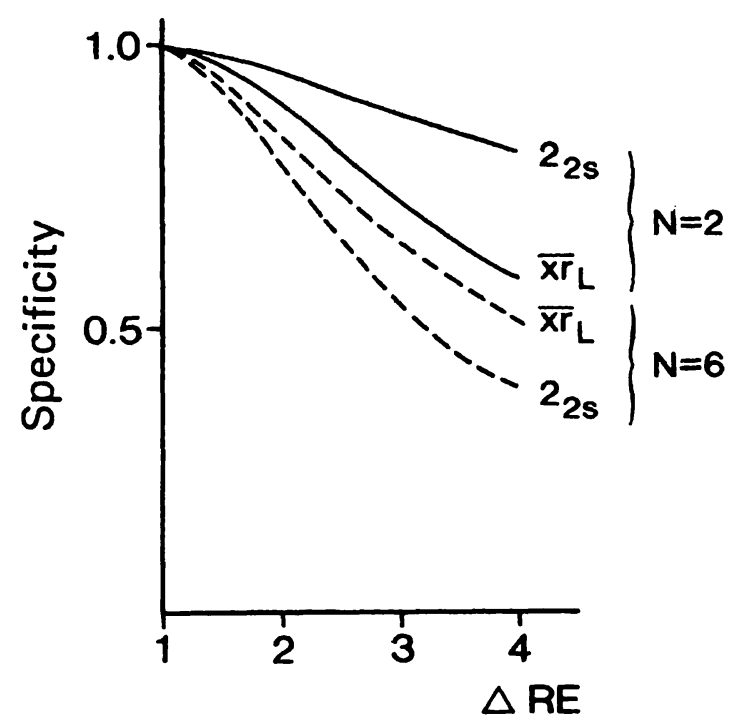

Fig. 6. Specificities of the 2 , and $\bar{x} \bar{r}_{L}$ rules as functions of the increased scatter.

$\Delta \mathrm{RE}$ is the increased scatter expressed as multiples of the standard deviation $s$.

The specificities of the $1_{3 s}$ and variance rules were evaluated (fig. 7), given equivalent type I errors $(0.00540$ for $N=2$ and 0.001609 for $N=6)$. The variance rule is almost totally specific, i.e. the frequency of false positives corresponds to the type I error. The $1_{3 s}$ rule, on the other side, has a very low specificity, which means that this rule really is an omnibus rule.

\section{Comparison of Powers of Rules Based on Control Observations from Several Runs}

In order to increase the power of detection of small, persistent location shifts, rules based on control observations from several runs were suggested in industrial quality control (7-9). E. g., a $\bar{x} \bar{r}_{L 3} / 2 \bar{x} \bar{r}_{L 2}$ (tab. 1) rule indicates rejection when the current mean deviates by more than $L 3$ from the target value, or when the means of the preceding and the current run both exceed the target value plus $L 2$, or both are below the target value minus $L 2 . L 2$ is usually assigned a value of $2 / 3 L 3$. This cumulating type of rule may be further extended to cover the latest five runs, i. e. $\bar{s}_{L 3} /$ $2 \bar{x} \bar{r}_{L 2} / 5 \bar{x} \bar{r}_{\bar{x}}$, where the lașt component indicates a rejection when five consecutive $\overline{\bar{x}} \overline{\dot{r}}$ values are either above or below the target value. Other types of rules based on runs tests etc. have also beẹn proposed $(8,9)$.

Westgard et al. (3) have modified these types of rules by using individual control values as a basis, e.g. $1_{3 s} /$ $2_{2 s} / R_{4 s} / 4_{1 s} / 10_{\bar{x}}$. This rule consists of one component directed towards both location shift and increased scatter $\left(1_{3 s}\right)$, one component responsive to increased

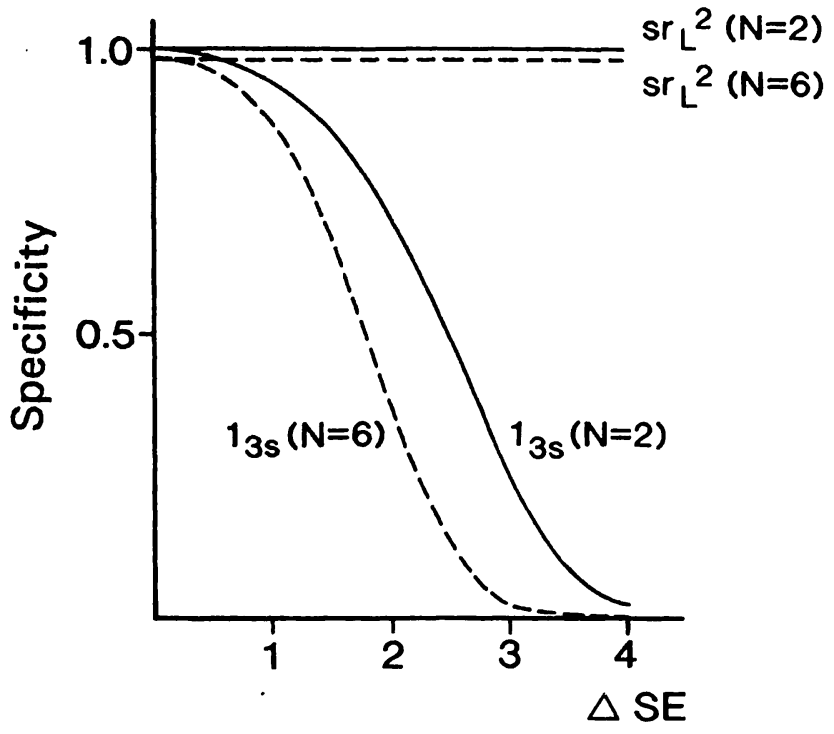

Fig. 7. Specificities of the $1_{3 s}$ and $s r_{L}^{2}$ rules as functions of the location shift.

$\Delta \mathrm{SE}$ is the location shift expressed in standard deviation $(s)$ units.

scatter $\left(\mathrm{R}_{4 s}\right)$, and three components that primarily react on location shift. Here the focus is on shift of location. Given $N=2$ controls per run, this rule covers the last five runs. The power is moderately smaller than that of the $\bar{x} \bar{r}_{L 3} / 2 \bar{x} \bar{r}_{L 2} / 5 \bar{x} \bar{r}_{\bar{x}}$ rule, which also covers five runs (fig. 8). It should be noted that for these types of rules, the power changes from the first to the fifth run of a new series, so the power should be interpreted as an average power obtained in simulation studies (Appendix). In this example, no between-

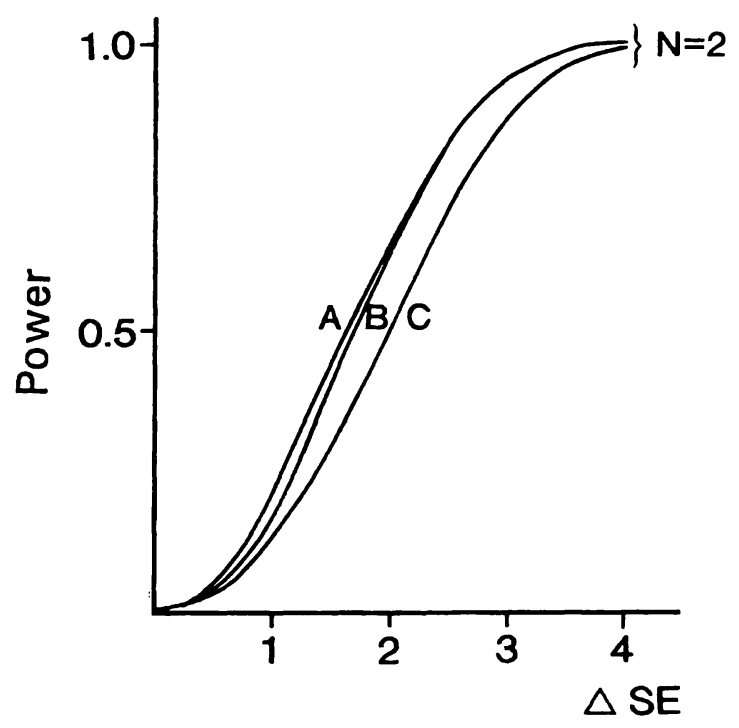

Fig. 8. Power curves for the moving-average rule $\left(\bar{x} \bar{r}_{L_{3}} / \bar{x} \bar{r}(5)_{L_{2}}\right)$, (A) the cumulative mean rule $\left(\bar{x} \bar{r}_{L 3} / 2 \bar{x}_{L_{L} /} / 5 \bar{x}_{\bar{x}}\right)$ (B), and the multi-rule $1_{3 s} / 2_{2 s} / R_{4 s} / 4_{1 s} / 10_{\dot{x}}(\mathrm{C})$.

$\Delta \mathrm{SE}$ is the location shift expressed in standard deviation (s) units. 
run component of variation has been assumed; inclusion of a between-run component of variance equal to the within-run component $\left(s^{2}\right)$ did not change the relation between the power curves.

With respect to location shifts, rules founded on the mean values are superior to individual value rules in all examples. When regarding several $(k)$ runs, one might suspect that a rule utilizing the mean of the current run and the overall mean of the $k$ latest runs might be even more effective than the rules regarded hitherto. Figure 8 also shows the power curve (A) for a $\bar{x} \bar{r}_{L 3} / \bar{x} \bar{r}(5)_{L 2}$ rule. A reject signal occurs when the current mean deviates by more than $L 3$ from the target value or when the overall mean of the five latest runs $(\bar{x} \bar{r}(5)$ ) deviates by more than $L 2$ (for run no. $2-4$ in a series, the overall mean is computed for the actual number of runs, and the $L 2$ limit is multiplied with the factor $\sqrt{5} / \sqrt{k}$ ). This moving-average rule (10) is slightly superior to the cumulative rule using $\bar{x} \bar{r}$ values.

Analogous to cumulating rules for detection of location shifts, cumulating variance rules can be constructed, e.g. a $s r_{L 3}^{2} / 2 s r_{L 2}^{2}$ rule, or a moving-variance rule, $s r_{L 3}^{2} / s r(5)_{L 2}^{2}$, where $s r(5)^{2}$ is the pooled within-run variance for the latest five runs. Such rules increase the power of detection of persistent increased scatter. Additionally, surveillance of the between-run component of variation might be considered on the basis of an analysis of components of variance model (11).

\section{Comparison of Robustness Towards Non-Normality of Mean and Individual Value Rules}

The type I error of the various quality control rules considered in previous sections are valid for Gaussian data distributions. When the distributions take nonnormal forms, the type I errors change more or less. If the relative change is small, a rule is said to be robust. Here the type I errors of various rules are compared for Gaussian and log-Gaussian data distributions (tab. 3). A log-Gaussian distribution of moderate skewness has been chosen (coefficient of skew-

Tab. 3. Relative changes of type I error when the distribution changes from a Gaussian to a log-Gaussian type with skewness 0.75 .

Number of controls

\begin{tabular}{lll}
\hline Rule & $N=2$ & $N=6$ \\
$\mathrm{l}_{3 s}$ & 3.0 & 3.0 \\
$1_{3 s} / 2_{2 s}$ & 2.6 & - \\
$1_{3 s} / 2_{2 s} / 4_{1 s} / 6_{\bar{x}}$ & - & 2.4 \\
$\bar{x} \bar{r}_{L}$ & 1.7 & 1.1 \\
\hline
\end{tabular}

ness 0.75 ; see figure 5 in 1.c. (12)). The type I errors of the individual value rules change with factors 3 $(N=2)$ to $2.4(N=6)$, whereas the relative changes are only $1.7(N=2)$ to $1.1(N=6)$ for the mean rule. The marked robustness of the mean rule for $N=6$ is a reflection of the central limit theorem, which says that the distribution of the mean converges towards a Gaussian distribution for increasing $N$, regardless of the type of distribution of the individual values. Robust control rules are preferable, because the robustness assures that the average run lengths for the in-control state are approximately valid, even for nonGaussian data distributions which occur frequently in clinical laboratories (13).

\section{Discussion}

Although most power comparisons for quality control rules are performed without standardization of type I errors, a few unbiased comparisons of mean rules with simple individual value rules have been undertaken (4). The power curves of the latter study clearly reveal the superiority of the mean rule for detection of a location shift. Apparently, similar systematic comparisons between the mean rule and multi-rules based on individual values have not been performed, which may explain the somewhat uncritical recommendation of multi-rules in the literature on quality control $(3,6)$. The advantage of the mean rule relies in the fact that the mean is the most effective measure of location. Theoretically, it can be proved that a statistical test based on the 'mean is the most powerful test for detection of a fixed shift of location (14). Heilbron et al. (15) addressed this point in the context of quality control, but little attention has been paid to their paper. In recent years, only a few authors $(16,17)$ have focused on mean and range rules for quality control in clinical chemistry.

Individual value control rules, as well as less complicated multi-rules, can be considered for manual analyses, because the relative loss of power or selectivity associated with these types of rules may be acceptable in view of their easier application. In the computerized laboratory, however, computation of mean and variance is no problem, and the gain in power or selectivity should be exploited. In particular, construction of multi-rules that are so complicated that computerized data treatment is necessary (18) seems inappropriate, because cumulating rules based on the mean are more simple and effective. Another possibility is cusum rules which also utilize quantitative test results effectively $(19,20)$. At a first glance, the recorded power differences for small to moderate errors may appear unimportant. However, a power increase from 
0.1 to 0.2 for a small systematic error means that the average number of runs before error is detected decreases from 10 to 5 . Mean and variance rules are also more easily designed to meet specified levels of type I error and power. Models that explicitly take a between-run component of variance into account can be devised (11). Extensive simulation studies to evaluate the performance are not necessary (21). Finally, the robustness of mean rules is an advantage.

When applying a mean rule to control values at several levels, e. g. two controls at each of two levels, one has to decide whether the control should be considered all together or separately for each level. In the former case, a constant shift over the entire range is most effectively detected, whereas the latter approach most easily reveals shifts restricted to a part of the range. When controls at several levels are compiled, and the analytical standard deviation $(s)$ varies with the level, a reasonable approach is to standardize the control measurements $x$ to $u_{i}=\left(x_{i}-\bar{x}\right) / s$. $u_{i}$ is distributed with mean zero and standard deviation of one when the analysis is in control. From the $u_{i}$ values, $\bar{x} \bar{r}$ is computed and plotted on a control chart with limits $\pm 31 / \sqrt{N}$. Similarly, $s r^{2}$ is computed and monitored. If there is only one control at each level, and a separate judgment is desired, the mean rule can apparently not be applied. It should be considered, however, that a moving-average rule for $N=1 \mathrm{con}$ trol per run is more effective than a multi-rule extending over several runs.

\section{Appendix}

Type I error and power of quality control rules

\section{Rules confined to a single run}

Assuming a Gaussian distribution, the type I error of the $1_{3 s}$ rule for $N=1$ is $\alpha=2 \cdot 0.00135=0.00270$.

\section{References}

1. Levey, S. \& Jennings, E. R. (1950) 'The Use of Control Charts in the Clinical Laboratory. Amer. J. Clin. Pathol. 20,1059-1066.

2. Henry, R. J. \& Segalove, M. (1952) The Running of Standards in Clinical Chemistry and the Use of the Control Chart. J. Clin. Pathol. 5, 305-311.

3. Westgard, J. O., Barry, P. L., Hurt, M. R. \& Groth, T. (1981) A Multi-Rule Shewhart Chart for Quality Control in Clinical Chemistry. Clin. Chem. 27, 493-501.

4. Westgard, J. O., Groth, T., Aronson, T., Falk, H. \& de Verdier, C.:H. (1977) Performance Characteristics of Rules for Internal Quality Control: Probabilities for False Rejection and Error Detection. Clin. Chem. 23, 1857-1867.

5. Westgard, J. O. \& Groth, T. (1979) Power Functions for Statistical Control Rules. Clin. Chem. 25, 863-869.
Given $N$ controls per run, all control observations are located within the control limits with a probability $(1-\alpha)^{N}$. Thus, the probability of observing at least one control value outside the limits (= type I error) is $1-(1-\alpha)^{N}$. E.g., $N=2$ yields a type I error of 0.0053927 , and $N=6$ results in a type I error of 0.0160910 . The power is calculated in an analogous manner.

For combined rules, the type I error and power are most easily determined by simulations. Simulating the null hypothesis, the frequency of rejections is the type I error. The power is obtained by simulation of alternative hypotheses. At least $10^{6}$ runs were simulated for estimation of type I errors, and $10^{4}$ runs for estimation of power.

Concerning the $\bar{x} \bar{r}_{L}$ rule, the type $I$ error is derived from the relation $L=z_{\alpha / 2} s / \sqrt{N}$, using a table of the normal distribution. The power for detection of a shift of location $\Delta$ is derived from:

$$
\mathrm{z}_{(1-\beta)}=(L-\Delta) /(s / \sqrt{N}) .
$$

Computation of type I error and power of the variance rule is decribed in 1.c. (11).

\section{Rules covering several runs}

For a quality control rule utilizing control observations from the latest $k$ runs, the probability of a reject signal increases from run number one to the $k$ th run (p. 105 in 1.c. (6)). A new cycle is started after each reject signal. By recording the frequency of rejections for a large number of simulated runs, the type I error and power are determined. These values should be regarded as average values. When comparing the power to different rules, reject limits have been adjusted so that the type I error of the first run and average type I error, respectively, are identical for the rules.

6. Westgard, J. O. \& Barry, P. L. (1986) Cost-Effective Quality Control: Managing the Quality and Productivity of Analytical Processes. Am. Assoc. for Clin. Chem. Washington, DC.

7. Page, E. S. (1955) Control Charts with Warning Lines. Biometrika 42, 243-254.

8. Nelson, L. S. (1984) The Shewhart Control Chart - Tests for Special Causes. J. Qual. Technol. 16, 237-239.

9. Nelson, L. S. (1985) Interpreting Shewhart X Control Charts. J. Qual. Technol. 17, 114-116.

10. Roberts, S. W. (1966) A Comparison of some Control Chart Procedures. Technometrics 8, 411-430.

11. Linnet, K. (1989) The Between-Run Component of Variation in Internal Quality Control. Clin. Chem. 35, 14161422. 
12. Westgard, J. O., Falk, H. \& Growth, T. (1979) Influence of a Between-Run Component of Variation, Choice of Control Limits, and Shape of Error Distribution on the Performance Characteristics of Rules for Internal Quality Control. Clin. Chem. 25, 394-400.

13. Passing, H. (1981) The Inadequacy of Normal Distribution Models for the Establishment of Assigned Values in Control Sera. J. Clin. Chem. Clin. Biochem. 19, 1145-1151.

14. Lehmann, E. (1959) Testing Statistical Hypotheses, Wiley, New York.

15. Heilbron, D. C., Eastman, J. W. \& Kelly, D. (1974) Warning Methods for Quality Control in Determination of Serum Calcium. Clin. Chem. 20, 1416-1421.

16. Saracci, R. (1974) The Power (Sensitivity) of Quality Control Plans in Clinical Chemistry. Amer. J. Clin. Pathol. 62, $398-406$.
17. Hainline, A. (1982) Quality Assurance: Theoretical and Practical Aspects. Selected Methods in Clin. Chem. 9, 17-31.

18. Blum, A. S. (1985) Computer Evaluation of Statistical Procedures, and a New Quality-Control Statistical Procedure. Clin. Chem. 31, 206-212.

19. Duncan, A. J. (1974) Quality Control and Industrial Statistics; 4th ed., Chapt. 22, R. D, Irwin, Inc., Homewood, II.

20. Westgard, J. O., Groth, T., Aronsson, T. \& de Verdier, CH. (1977) Combined Shewhart-Cusum Control Chart for Improved Quality Control in Clinical Chemistry. Clin. Chem. 23, $1881-1887$.

21. Westgard, J. O. \& Groth, T. (1981) Design and Evaluation of Statistical Control Procedures: Applications of a Computer "Quality Control Simulator" Program. Clin. Chem. 27, 1536-1545.

Kristian Linnet, M.D.

The Department of Clin. Chem. KK 7642

Rigshospitalet

DK-2100 Copenhagen 